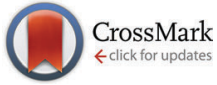

Cite this: Phys. Chem. Chem. Phys., 2015, 17, 10348

Received 12th February 2015, Accepted 23rd March 2015

DOI: $10.1039 / c 5 c p 00910 c$

www.rsc.org/pccp

\title{
Adsorption of polyelectrolytes to like-charged substrates induced by multivalent counterions as exemplified by poly(styrene sulfonate) and silica $\dagger$
}

\author{
Alberto Tiraferri, ${ }^{a b}$ Plinio Maroni ${ }^{\mathrm{b}}$ and Michal Borkovec ${ }^{\text {b }}$
}

\begin{abstract}
The present study demonstrates that multivalent counterions trigger adsorption of polyelectrolytes on a like-charged substrate. In particular, adsorption of polystyrene sulfonate on silica is studied experimentally in $\mathrm{NaCl}, \mathrm{MgCl}_{2}$, and $\mathrm{LaCl}_{3}$ solutions by optical reflectivity. While adsorption is negligible in the presence of $\mathrm{Na}^{+}$, the polyelectrolyte adsorbs in the presence of $\mathrm{Mg}^{2+}$ and $\mathrm{La}^{3+}$. The adsorbed amount of the polyelectrolyte goes through a maximum as a function of the salt concentration. This maximum increases with increasing valence and shifts to lower salt concentrations. At low salt concentration, the adsorption is negligible. At intermediate salt level, ripening and multilayer formation leads to continuous growth of the adsorbed layer. At higher salt level, blocking and formation of a monolayer lead to saturation. These results are tentatively interpreted in terms of a charge reversal of the polyelectrolyte-metal complex. The molecular mass of the polyelectrolyte has an important effect on the adsorption behavior, whereby the tendency towards ripening becomes more pronounced at large molecular mass.
\end{abstract}

Polyelectrolytes adsorb readily to oppositely charged substrates, and this process is exploited in various applications, including water treatment, papermaking, formulation of foods, or multilayer coatings. ${ }^{1-7}$ The adsorption process is mainly driven electrostatically. Thereby, attraction between the polyelectrolyte and the oppositely charged substrate induces rapid formation of the adsorbed layer, while repulsion between the equally charged polyelectrolyte segments leads to surface saturation and blocking.

Given the importance of electrostatic interactions in the adsorption process, one might be tempted to think that polyelectrolytes should not adsorb on like-charged substrates. However, numerous applications rely on polyelectrolytes carrying the same charge as the substrates, for example, DNA imaging, water

\footnotetext{
${ }^{a}$ Department of Environment, Land, and Infrastructure Engineering;

Polytechnic University of Turin, Torino, Italy

${ }^{b}$ Department of Inorganic and Analytical Chemistry, University of Geneva,

Sciences II, 30 Quai Ernest-Ansermet, 1205 Geneva, Switzerland.

E-mail: michal.borkovec@unige.ch

$\dagger$ Electronic supplementary information (ESI) available: Experimental details and additional adsorption traces. See DOI: 10.1039/c5cp00910c
}

treatment, or formulation of particle slurries. ${ }^{7-9}$ Moreover, several studies suggest that polyelectrolytes actually do adsorb to likecharged substrates. ${ }^{9-16}$ In such situations, salts containing multivalent cations are often added (e.g., $\left.\mathrm{Ca}^{2+}, \mathrm{Ni}^{2+}, \mathrm{Fe}^{3+}, \mathrm{Al}^{3+}\right) .^{7,12,13}$ Specific interaction of polyacrylates with calcite might involve similar processes, since their adsorption leads to calcite dissolution and release of $\mathrm{Ca}^{2+}$ ions. ${ }^{17}$

A potential like-charge adsorption mechanism stresses the importance of non-electrostatic forces, such as van der Waals or hydrophobic interactions. ${ }^{15,16,18-20}$ This mechanism seems particularly pronounced for comb-like or block-copolymers, whereby the hydrophobic part of the polymer is mainly responsible for the adsorption. An alternative mechanism came to focus recently, especially in the theoretical community. Computational studies suggest that polyelectrolytes adsorb to like-charged substrates through electrostatic forces only. ${ }^{18,21-23}$ A simplified interpretation of these findings involves a charge reversal through the binding of multivalent ions to the substrate or the polyelectrolyte. This situation may again lead to electrostatically induced adsorption, similar to the case of oppositely charged polyelectrolytes.

The effect of multivalent ions on polyelectrolyte adsorption to like-charged substrates was so far only studied for DNA and mica in detail. ${ }^{9-11}$ Imaging by atomic force microscopy (AFM) was used to demonstrate that anionic DNA does not adsorb to negatively charged mica in monovalent salt solutions, but that adsorption occurs from solutions containing divalent and trivalent cations. AFM was also used to show that $\mathrm{Mg}^{2+}$ ions induce adsorption of poly(styrene sulfonate) (PSS) on mica. ${ }^{16}$ Classical batch experiments also suggest that $\mathrm{Ca}^{2+}$ and $\mathrm{Mg}^{2+}$ ions enhance the adsorption of anionic polyacrylates to negatively charged alumina and titania particles. ${ }^{12,13}$ With the exception of DNA, however, the available experimental data base remains weak.

For these reasons, we report experimental findings demonstrating that multivalent counterions strongly promote adsorption of negatively charged PSS on like-charged silica in aqueous solutions. Our technique of choice is optical reflectivity, ${ }^{4,5}$ as it permits to study in real time adsorption to a planar substrate 
(1)
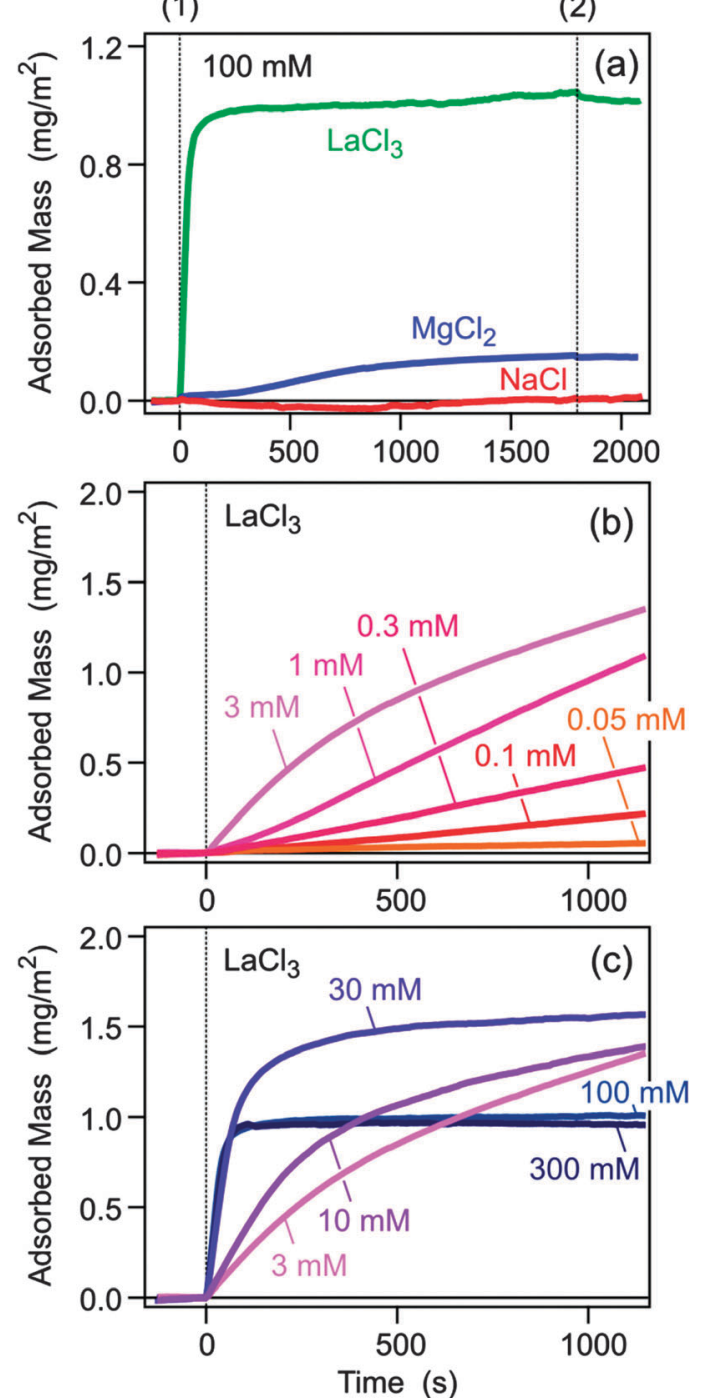

Fig. 1 Adsorbed mass of negatively charged polyelectrolyte PSS on likecharged silica substrate in various electrolytes measured by optical reflectivity. The substrate is first rinsed with pure electrolyte solution and then the PSS solution is injected at time (1). The PSS concentration is $10 \mathrm{mg} \mathrm{L}^{-1}$ and solution $\mathrm{pH}$ 6.0. Molecular mass of PSS is $30 \mathrm{~kg} \mathrm{~mol}^{-1}$ unless indicated otherwise. (a) Adsorption in electrolyte solution with various counterions $\mathrm{Na}^{+}, \mathrm{Mg}^{2+}$, and $\mathrm{La}^{3+}$ at a concentration of $100 \mathrm{mM}$. At time (2) the substrate was flushed with pure electrolyte solution. Adsorption traces for (b) low and (c) high $\mathrm{LaCl}_{3}$ concentrations. The different concentrations are labeled.

in situ in the impinging-jet geometry with excellent sensitivity down to few $\mu \mathrm{g} \mathrm{m}^{-2}$. Experimental details are given in the ESI. $\dagger$

The silica substrate was first flushed with pure electrolyte solution of $\mathrm{pH} 6.0$, and subsequently a solution of the sodium salt of PSS of a molecular mass of $30 \mathrm{~kg} \mathrm{~mol}^{-1}$ of a concentration of $10 \mathrm{mg} \mathrm{L}^{-1}$ in the same electrolyte of the same $\mathrm{pH}$ was injected. After a period of time, the substrate was again rinsed by the pure electrolyte solution. The recorded traces of the adsorbed amount are shown in Fig. 1a. As one expects, there is very little adsorption in the presence of $\mathrm{Na}^{+}$. Adsorption already occurs in the presence of $\mathrm{Mg}^{2+}$, while for $\mathrm{La}^{3+}$ the adsorbed amount is substantial. The latter two ions were chosen due to
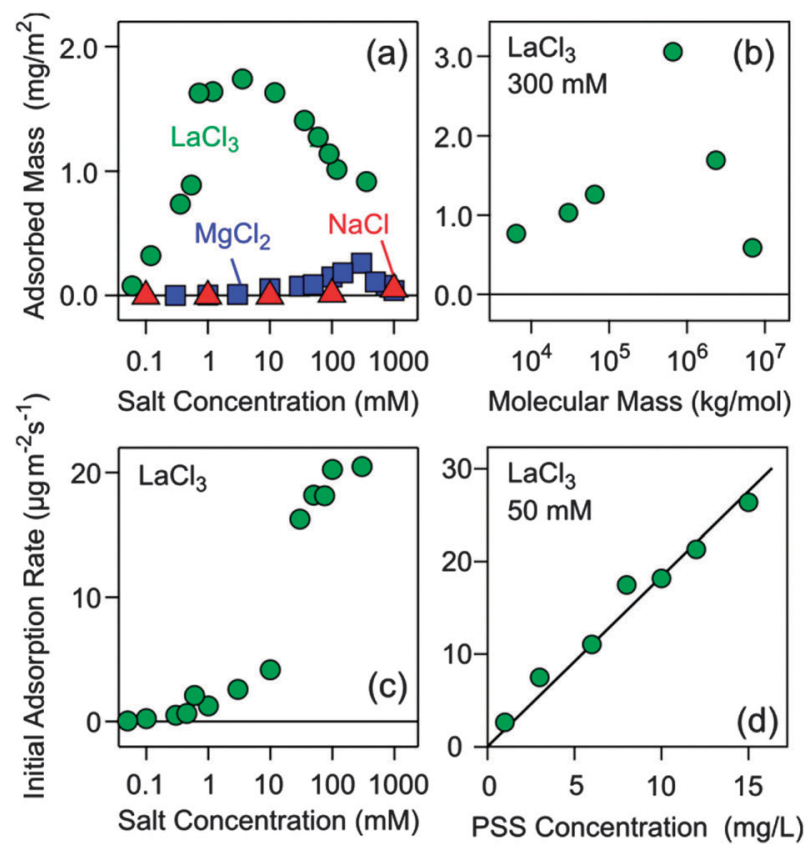

Fig. 2 Adsorbed mass and initial adsorption rates for PSS on like-charged silica at $\mathrm{pH}$ 6.0. The PSS concentration is $10 \mathrm{mg} \mathrm{L}^{-1}$ and the molecular mass of PSS is $30 \mathrm{~kg} \mathrm{~mol}^{-1}$, unless indicated otherwise. (a) Amount adsorbed after $30 \mathrm{~min}$ and subsequent desorption during $10 \mathrm{~min}$ with the same electrolyte solution. (b) Adsorbed mass after 30 min versus the molecular mass. Initial adsorption rate versus (c) the concentration of the $\mathrm{LaCl}_{3}$ salt and (d) the polyelectrolyte concentration in $50 \mathrm{mM} \mathrm{LaCl}_{3}$ solution.

negligible hydrolysis. ${ }^{24}$ Appreciable concentrations of hydroxide complexes $\mathrm{MgOH}^{+}$form above $\mathrm{pH} 10$, and $\mathrm{LaOH}^{2+}$ above $\mathrm{pH} 8$. After rinsing the adsorbed layer with pure electrolyte solution, only minor desorption could be evidenced.

The adsorbed amount was studied for different salt concentrations, and the result is shown in Fig. 2a. In the presence of monovalent counterions $\mathrm{Na}^{+}$, adsorption remains negligible. For $\mathrm{Mg}^{2+}$ one finds a maximum adsorbed amount of $0.2 \mathrm{mg} \mathrm{m}^{-2}$ at a salt level around $300 \mathrm{mM}$, while for $\mathrm{La}^{3+}$ the maximum exceeds $1.5 \mathrm{mg} \mathrm{m}^{-2}$ and shifts to lower concentrations around $3 \mathrm{mM}$. The present data suggest that the adsorbed amount goes through a maximum, and that its position decreases with the valence of the counterion. A similar maximum was predicted by computer simulations for the adsorption of polyacrylates to silica in the presence of $\mathrm{Ca}^{2+}$ ions, albeit at lower salt concentrations. ${ }^{18}$

To obtain better insight into the adsorption mechanism of PSS to silica in the presence of $\mathrm{La}^{3+}$, a detailed kinetic study was carried out. The initial adsorption rates were also studied. ${ }^{4}$ The respective traces are shown in Fig. $1 \mathrm{~b}$ and $\mathrm{c}$ and the corresponding initial adsorption rates in Fig. 2c. At a concentration of $\mathrm{La}^{3+}<0.1 \mathrm{mM}$, the adsorption is negligible. However, the adsorbed amount increases quickly with increasing concentration. While the initial adsorption rate remains low, the adsorbed amount starts to increase after an initial induction period. More surprisingly, however, the adsorbed amount continues to increase, without showing any signs of saturation. In some experiments, adsorbed amounts even exceed about $10 \mathrm{mg} \mathrm{m}^{-2}$, but these conditions were not analyzed in detail due to eventual 
non-linearity of the detector. This situation indicates that the initial adsorption step of the polyelectrolyte to the surface is unfavorable, but once few polyelectrolyte chains are adsorbed, they serve as nucleating sites for further adsorption. In this regime, the surface undergoes ripening, whereby a polyelectrolyte multilayer is being formed.

A saturation of the surface sets in about at concentrations $>3 \mathrm{mM}$, and this blocking process becomes fully evident at higher salt concentrations. The observed saturation amount of about $1.0 \mathrm{mg} \mathrm{m}^{-2}$ roughly corresponds to a dense polyelectrolyte monolayer. In this regime, the initial adsorption kinetics is fast and first order with respect to the polymer concentration (Fig. 2d). The resulting adsorption rate coefficient is $1.8 \mu \mathrm{m} \mathrm{s}^{-1}$. When one assumes perfect sink conditions at the surface, one can evaluate the transport controlled rate coefficient of the impinging-jet geometry. ${ }^{4,26}$ By approximating the diffusion coefficient of PSS to be comparable to the one in a monovalent salt solution of the same ionic strength, ${ }^{25}$ one obtains a sticking coefficient of about 0.2. These features resemble adsorption of polyelectrolytes to oppositely charged substrates. ${ }^{3,4}$

The molecular mass of the polyelectrolyte has an important effect on the adsorption of PSS on silica as induced by $\mathrm{La}^{3+}$ ions. This effect was studied in $300 \mathrm{mM} \mathrm{LaCl}_{3}$ solutions. As illustrated in Fig. 2b, the adsorbed amount goes through a maximum around $3.2 \mathrm{mg} \mathrm{m} \mathrm{m}^{-2}$ near $700 \mathrm{~kg} \mathrm{~mol}^{-1}$. At low molecular mass, the adsorption saturates, while at higher molecular mass, a gradual increase of the adsorbed amount can be evidenced without a clear onset of saturation. The actual adsorption traces shown in the supplement suggest that ripening becomes more important with increasing molecular mass.

We propose the following tentative interpretation of these results. PSS is a highly charged and hydrophobic polyelectrolyte, and therefore we suspect that it binds $\mathrm{La}^{3+}$ ions, forming a strong metal-polyelectrolyte complex. At low $\mathrm{La}^{3+}$ concentrations, the number of the bound $\mathrm{La}^{3+}$ ions is small and the polyelectrolytemetal complex remains negatively charged. With increasing $\mathrm{La}^{3+}$ concentrations, however, the number of bound $\mathrm{La}^{3+}$ ions increases. At a particular $\mathrm{La}^{3+}$ concentration, the polyelectrolyte-metal complex will be neutral, probably around $1 \mathrm{mM}$. Increasing the $\mathrm{La}^{3+}$ concentration further, additional $\mathrm{La}^{3+}$ ions are bound by the polyelectrolyte, leading to a positively charged metal-polyelectrolyte complex. The fact that such a charge reversal might occur was also suggested by computer simulations. ${ }^{27,28}$ This charge reversal is also consistent with the observed precipitation of PSS by $\mathrm{La}^{3+}$ ions, which occurs at intermediate $\mathrm{La}^{3+}$ concentrations, while at low and high concentrations, the polyelectrolyte remains soluble. ${ }^{29,30}$ These precipitation experiments were carried out at much higher PSS concentrations than the ones used for the present experiments. We have no evidence of precipitation in the PSS solutions prepared here. On the other hand, we surmise that silica substrate remains negative. Divalent ions were shown to lead to a weak charge reversal of silica in weakly basic solutions at very high salt concentrations. ${ }^{31}$ Silica is less charged at the $\mathrm{pH}$ used suggesting that charge reversal of the substrate by $\mathrm{La}^{3+}$ is unlikely.

Based on this charge reversal scenario, the observed results can be interpreted as follows (see Fig. 3a-c). At low $\mathrm{La}^{3+}$

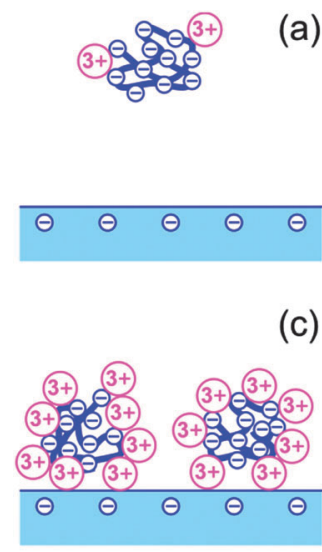

(b)

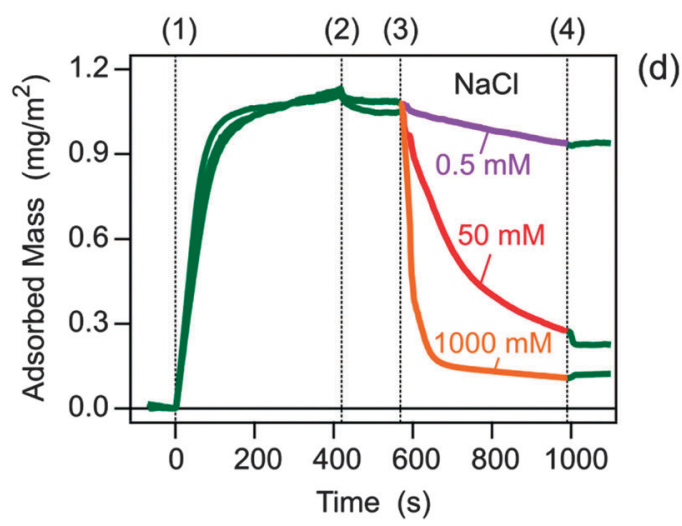

Fig. 3 Proposed mechanism of the polyelectrolyte adsorption on likecharge substrates mediated by multivalent counterions. (a) No adsorption at low salt concentration, (b) ripening involving multilayer formation at intermediate concentrations, and (c) monolayer formation and blocking at high salt concentration. (d) Adsorption of PSS in $\mathrm{LaCl}_{3}$ solution and desorption with $\mathrm{NaCl}$ at $\mathrm{pH}$ 6.0. The substrate is flushed with $50 \mathrm{mM}$ $\mathrm{LaCl}_{3}$ solution, then at time (1) PSS solution of $10 \mathrm{mg} \mathrm{L}^{-1}$ in the same $\mathrm{LaCl}_{3}$ solution is introduced, at time (2) the substrate is rinsed with pure $\mathrm{LaCl}_{3}$ solution, at time (3) a pure $\mathrm{NaCl}$ solution of the indicated concentration is introduced, and at time (4) the substrate is finally rinsed with pure $\mathrm{LaCl}_{3}$ solution. The different traces shown between (1) and (3) are repetitions of the same adsorption experiment, and illustrate the good reproducibility of these experiments.

concentrations, the negatively charged metal-polyelectrolyte complex does not adsorb to the negatively charged substrate due to electrostatic repulsion. At intermediate $\mathrm{La}^{3+}$ concentrations around $1 \mathrm{mM}$, the hydrophobic forces acting between the neutral polyelectrolyte-metal complexes induce a continuous surface deposition, leading to ripening, and the formation of a thick multilayer. At higher $\mathrm{La}^{3+}$ concentrations, the positively charged polyelectrolyte-metal complex adsorbs rapidly due to electrostatic attraction to the negatively charged silica surface. The adsorption process continues until the electrostatic repulsion between the adsorbed complexes becomes sufficiently important such that the surface saturates. At this point, the adsorbed polyelectrolyte monolayer develops a positive charge, which prevents further adsorption of the positively charged polyelectrolyte-metal complexes. The latter situation resembles the adsorption of a polyelectrolyte to an oppositely charged substrate. While the present interpretation in terms of the 
charge reversal of the polyelectrolyte seems suggestive to us, other possible mechanisms may include aggregation of polyelectrolytes in solution, surface induced precipitation, or contributions of hydrophobic interactions.

The fact that this mechanism based on the charge reversal is incomplete is further underlined by the non-monotonous molecular mass dependence discussed above (Fig. 2b). While the increase in the adsorbed mass is consistent with the initial increase in size of the polyelectrolyte-metal complex, the decrease at larger molecular mass and the tendency of the adsorbed layer towards ripening suggests the possible onset of different processes. They could involve the formation of pearlnecklace structures within the polyelectrolyte-metal complex or the formation of polyelectrolyte aggregates in solution mentioned above. $^{32,33}$ The deposition of such aggregates may lead to the observed ripening of the adsorbed layer. The formation of these structures could be mediated by complexation of multivalent ions by the polyelectrolytes, and could resemble the effect on the aggregation of colloidal particles due to charge reversal induced by adsorbing polyelectrolytes or multivalent ions. $^{3,34}$ The presently proposed adsorption mechanism remains speculative for the moment.

We remarked that rinsing the layer with the pure electrolyte solution containing multivalent ions does not lead to any appreciable polyelectrolyte desorption, which indicates irreversible adsorption within the experimental time window. However, desorption can be readily induced by rinsing with a solution containing monovalent counterions (Fig. 3d). Desorption becomes increasingly rapid with increasing concentration of $\mathrm{NaCl}$, indicating that bound $\mathrm{La}^{3+}$ in the adsorbed layer is exchanged with $\mathrm{Na}^{+}$, and the resulting polyelectrolyte layer containing $\mathrm{Na}^{+}$becomes unstable and desorbs. Desorption process can be stopped by reintroducing the $\mathrm{LaCl}_{3}$ solution, whereby $\mathrm{Na}^{+}$ions are exchanged back with $\mathrm{La}^{3+}$ ions.

To conclude, we provide experimental evidence with optical reflectivity that highly charged polyelectrolytes adsorb to a likecharged substrate in the presence of multivalent counterions. The adsorbed amount of the polyelectrolyte versus the salt concentration goes through a maximum, which becomes higher with increasing valence and shifts to lower concentrations. At low concentration of multivalent ions, no adsorption is observed. At intermediate ionic levels, the adsorbed layer forms slowly, but continues to grow due to ripening and multilayer formation. At higher ionic levels, the adsorption saturates, probably leading to blocking and formation of a monolayer. The tendency towards ripening increases with increasing molecular mass of the polyelectrolyte. A tentative interpretation of these findings is based on the formation of a polyelectrolyte-metal complex between the anionic polymer chains and the multivalent cations, which undergoes a charge neutralization and subsequent charge reversal. Since computer simulations have shown that such adsorption of polyelectrolytes to like-charged substrates to be governed by electrostatic forces only, we suspect that the present findings could be generic and possibly applicable to a wide variety of systems. Specific interactions may of course modify the behavior of individual systems, for example, hydrophobic interactions may act together with the electrostatic interactions discussed here. While the extension of the present results to the practically more relevant multivalent cations $\mathrm{Fe}^{3+}$ and $\mathrm{Al}^{3+}$ would be important, such systems are probably complicated by the extensive hydrolysis reactions of these ions. ${ }^{24}$

\section{Acknowledgements}

This work was supported by a Marie Curie Intra-European Fellowship to A.T. within the Seventh European Community Framework Programme (PIEF-GA-2012-327977), by the Swiss National Science Foundation, and by the University of Geneva.

\section{References}

1 R. Mezzenga, P. Schurtenberger, A. Burbidge and M. Michel, Nat. Mater., 2005, 4, 729-740.

2 A. Martin-Molina, G. Luque-Caballero, J. Faraudo, M. QuesadaPerez and J. Maldonado-Valderrama, Adv. Colloid Interface Sci., 2014, 206, 172-185.

3 I. Szilagyi, G. Trefalt, A. Tiraferri and M. Borkovec, Soft Matter, 2014, 10, 2479-2502.

4 I. Popa, B. P. Cahill, P. Maroni, G. Papastavrou and M. Borkovec, J. Colloid Interface Sci., 2007, 309, 28-35.

5 A. Tiraferri, P. Maroni, D. C. Rodriguez and M. Borkovec, Langmuir, 2014, 30, 4980-4988.

6 K. L. Chen and M. Elimelech, Environ. Sci. Technol., 2008, 42, 7607-7614.

7 B. Bolto and J. Gregory, Water Res., 2007, 41, 2301-2324.

8 B. J. Lee, M. A. Schlautman, E. Toorman and M. Fettweis, Water Res., 2012, 46, 5696-5706.

9 O. Pietrement, D. Pastre, S. Fusil, J. Jeusset, M. O. David, F. Landousy, L. Hamon, A. Zozime and E. Le Cam, Langmuir, 2003, 19, 2536-2539.

10 D. Pastre, L. Hamon, F. Landousy, I. Sorel, M. O. David, A. Zozime, E. Le Cam and O. Pietrement, Langmuir, 2006, 22, 6651-6660.

11 C. Rivetti, M. Guthold and C. Bustamante, J. Mol. Biol., 1996, 264, 919-932.

12 L. Dupont, A. Foissy, R. Mercier and B. Mottet, J. Colloid Interface Sci., 1993, 161, 455-464.

13 J. Sun, L. Bergstrom and L. Gao, J. Am. Ceram. Soc., 2001, 84, 2710-2712.

14 N. G. Hoogeveen, M. A. Cohen Stuart and G. J. Fleer, J. Colloid Interface Sci., 1996, 182, 133-145.

15 P. Maroni, F. J. Montes Ruiz-Cabello and A. Tiraferri, Soft Matter, 2014, 10, 9220-9225.

16 A. Gromer, M. Rawiso and M. Maaloum, Langmuir, 2008, 24, 8950-8953.

17 C. Geffroy, J. Persello, A. Foissy, B. Cabane and F. Tournilhac, Rev. Inst. Fr. Pet., 1997, 52, 183-190.

18 M. Turesson, C. Labbez and A. Nonat, Langmuir, 2011, 27, 13572-13581.

19 T. Abraham, D. Christendat, Z. Xu, J. Masliyah, J. F. Gohy and R. Jerome, AIChE J., 2004, 50, 2613-2626. 
20 C. P. Whitby, P. J. Scales, F. Grieser, T. W. Healy, G. Kirby, J. A. Lewis and C. F. Zukoski, J. Colloid Interface Sci., 2003, 262, 274-281.

21 R. Messina, C. Holm and K. Kremer, J. Chem. Phys., 2002, 117, 2947-2960.

22 L. Wang, H. J. Liang and J. Z. Wu, J. Chem. Phys., 2010, 133, 044906.

23 G. Luque-Caballero, A. Martin-Molina and M. QuesadaPerez, J. Chem. Phys., 2014, 140, 174701.

24 C. F. Baes and R. E. Mesmer, The Hydrolysis of Cations, Krieger Publishing, Malabar, 1976.

25 N. Borochov and H. Eisenberg, Macromolecules, 1994, 27, 1440-1445.

26 Z. Adamczyk, B. Siwek and P. Warszynski, J. Colloid Interface Sci., 2002, 248, 244-254.
27 M. Deserno, F. Jimenez-Angeles, C. Holm and M. LozadaCassou, J. Phys. Chem. B, 2001, 105, 10983-10991.

28 K. M. Wu, Y. F. Wei and P. Y. Hsiao, Electrophoresis, 2011, 32, 3348-3363.

29 M. O. de la Cruz, L. Belloni, M. Delsanti, J. P. Dalbiez, O. Spalla and M. Drifford, J. Chem. Phys., 1995, 103, 5781-5791.

30 I. Sabbagh and M. Delsanti, Eur. Phys. J. E: Soft Matter Biol. Phys., 2000, 1, 75-86.

31 F. H. J. van der Heyden, D. Stein, K. Besteman, S. G. Lemay and C. Dekker, Phys. Rev. Lett., 2006, 96, 224502.

32 J. Jeon and A. V. Dobrynin, Macromolecules, 2007, 40, 7695-7706.

33 M. O. Khan and B. Jonsson, Biopolymers, 1999, 49, 121-125.

34 P. Sinha, I. Szilagyi, F. J. Montes Ruiz-Cabello, P. Maroni and M. Borkovec, J. Phys. Chem. Lett., 2013, 4, 648-652. 